\title{
The role of the adipose tissue-derived mesenchymal stem cells enriched with melatonin in pancreatic cellular regeneration
}

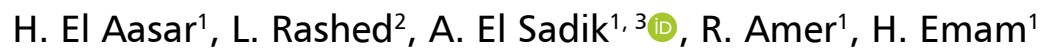 \\ ${ }^{1}$ Department of Anatomy and Embryology, Faculty of Medicine, Cairo University, Cairo, Egypt \\ ${ }^{2}$ Department of Biochemistry and Molecular Biology, Faculty of Medicine, Cairo University, Cairo, Egypt \\ ${ }^{3}$ Department of Anatomy and Histology, College of Medicine, Qassim University, Buraidah, Saudi Arabia
}

[Received: 5 July 2021; Accepted: 9 September 2021; Early publication date: 15 September 2021]

Background: Adipose tissue-derived mesenchymal stem cells (AD-MSCS) were proved to differentiate into insulin-producing cells (IPCS), but the amount of insulin secreted was relatively low compared to the insulin secreted by mature pancreatic islets. Enrichment of MSCs culture with melatonin (MT) was found to promote cartilage matrix synthesis, osteogenic and neuronal differentiation. Therefore, the present study was conducted to evaluate the potential role of MT pre-treated AD-MSCs in enhancing the treatment and regeneration of the islet cells of Langerhans in rats with diabetes induced by streptozotocin (STZ).

Materials and methods: Forty adult male Sprague Dawley albino rats were divided equally into groups; group I (control group), group II (STZ group), group III (STZ + AD-MSCS) and group IV (STZ+MT pre-treated AD-MSCS). Biochemical studies were implemented including measurements of the body weight, fasting blood glucose and serum insulin levels, Interleukin 17 (IL-17) and IL-10. Samples of the pancreas were taken and prepared for light, fluorescent microscopic examination, proliferating cell nuclear antigen and caspase-3 immunohistochemical studies and histomorphometric analysis.

Results: The present study confirmed the regenerative and therapeutic effects of AD-MSCs on the pancreatic cells. Concomitant supply of MT to the culture of $A D-M S C S$, in group IV, was shown to retain the normal architecture of the islet cells of Langerhans. They appeared well-defined and lightly stained, surrounded by classical pancreatic acini and contained a large number of islet cells with vesicular nuclei and prominent nucleoli. Improvement of all the biochemical parameters, in the same group, was demonstrated by increased body weight and serum insulin levels with a decrease in the fasting blood glucose levels. Significant decrease in the pro-inflammatory cytokine; IL-17 and increase in the anti-inflammatory cytokine; IL-10, compared to the STZ group, were also discovered. Significant increase in the proliferating cell nuclear antigen proliferation index, decrease in caspase-3 and increase in PKH26 labelled MSCs area per cent was recorded in the group of AD-MSCs enriched with MT compared to the group of AD-MSCS without MT. Conclusions: The present study confirmed the potential therapeutic and protective role of MT pre-treated AD-MSCS against the STZ-induced pancreatic islet cells'

Address for correspondence: Dr. A. El Sadik, Department of Anatomy and Histology, College of Medicine, Qassim University, P.O. Box 6655, Buraidah 51452, Kingdom of Saudi Arabia, e-mail: abeer.ouaida@kasralainy.edu.eg

This article is available in open access under Creative Common Attribution-Non-Commercial-No Derivatives 4.0 International (CC BY-NC-ND 4.0) license, allowing to download articles and share them with others as long as they credit the authors and the publisher, but without permission to change them in any way or use them commercially. 
damage. Further studies are recommended to investigate the efficacy of MT and AD-MSCs over longer experimental durations. (Folia Morphol 2022; 81, 4: 931-941)

Key words: melatonin, adipose tissue-derived mesenchymal stem cells, islets of Langerhans, diabetes; interleukin 17, interleukin 10

\section{INTRODUCTION}

Remarkable advancement has been achieved concerning the generation of functional pancreatic $\beta$-cells from the mesenchymal stem cells (MSCs). Recently, marked efforts have been accomplished to generate single hormone-positive $\beta$-cells capable of glucose-stimulated insulin secretion (GSIS). These achievements were focused on generating fully functional $\beta$ and islet cells that could soon replace the human islets as a source of insulin-producing cells (IPCs) [18]. Obtaining MSCs faces practical limitations, concerning the difficulty and invasiveness of the process. The difficulty of isolating the samples and the potential adverse effects of harvesting the cells from the donor must be considered. Isolating them from bone marrow, for instance, can result in pain, bleeding or infection, thus making harvesting MSCs from this source more problematic than harvesting them from peripheral blood or surgical remnants such as adipose tissue [3].

Moshtagh et al. [14] used adipose tissue-derived mesenchymal stem cells (AD-MSCs) to produce IPCs. The resultant cells stained positive for dithizone and produced insulin when transplanted into streptozotocin (STZ)-induced diabetic mice, but the amount of insulin secreted was relatively low compared to the insulin secreted by mature pancreatic islets. Karaoz et al. [11] demonstrated that AD-MSCs differentiated into IPCs after 38 days' co-culture with islet cells. Insulin and C-peptide production were confirmed by enzyme-linked immunosorbent assay (ELISA) and immunostaining. After co-transplantation of IPCs and islet cells under the kidney capsule, the hyperglycaemic state was normalised in diabetic rats. Moreover, it was revealed that the combination of differentiated ADMSCs and islet cells resulted in better recovery from diabetes compared to islets transplanted alone or co-transplantation of islets with differentiated bone marrow (BM)-MSCs [8]. Thakkar et al. [21] showed that AD-MSCs displayed the ability to lessen the hyperglycaemia in diabetic patients. Human AD-MSCs were proved to differentiate into IPCs, confirmed by the expression of immunofluorescent pancreatic markers, such as paired box protein (Pax6), pancreatic duodenal homeobox-1 (PDX-1), insulin gene enhancer-1 (ISL-1) and insulin promoter factor-1 (Ipf-1). The resultant IPCs were injected into the portal circulation resulting in significant improvement in the plasma glucose and glycated haemoglobin (HbA1c) levels, augmented C-peptide levels and decreased exogenous insulin requirement.

Melatonin (MT) or N-acetyl-5-methoxytryptamine is the prime hormone secreted by the pineal gland [19]. Enrichment of MSCs culture with MT was found to enhance cartilage matrix synthesis as well as the expression of chondrogenic differentiation markers of human mesenchymal stem cells (hMSCs) [17]. It also improved the osteogenic differentiation of hMSCs, shown by an increase in the expression of alkaline phosphatases, osteocalcin, osteopontin and bone sialoprotein in hMSCs cultures [12]. MT also promoted neuronal differentiation of MSCs and stimulated neural stem cells to differentiate into dopaminergic neurons in rats [10]. On the other hand, MT was reported to inhibit adipogenesis and adipogenic differentiation of MSCs [2]. Hu and Li [9] demonstrated that the excessive reactive oxygen species (ROS) in MSCs cultures in vitro and the injured microenvironment in vivo significantly reduce the therapeutic effects of MSCs. They added that MT downregulated the expression of ROS generating genes, reduced the release of inflammatory cytokines, enhanced the proliferation capacity of MSCs and significantly reduced the apoptosis of MSCs in vitro and in vivo. Therefore, the aim of the present study was to determine the potential role of MT pre-treated AD-MSCs in promoting the regeneration of islets of Langerhans in diabetic rats induced by STZ.

\section{MATERIALS AND METHODS}

Isolation, propagation and identification of MSCs

Abdominal adipose tissue was taken from Sprague Dawley albino rats after sacrificing. Extracellular matrix was digested with $0.075 \%$ type I collagenase $\left(37^{\circ} \mathrm{C}\right.$ and $5 \% \mathrm{CO}_{2}$ for $30 \mathrm{~min}$ ) and centrifuged at $500 \mathrm{~g}$ for $5 \mathrm{~min}$; then the pellet was cultured in high glucose Dulbecco's modified Eagle's 

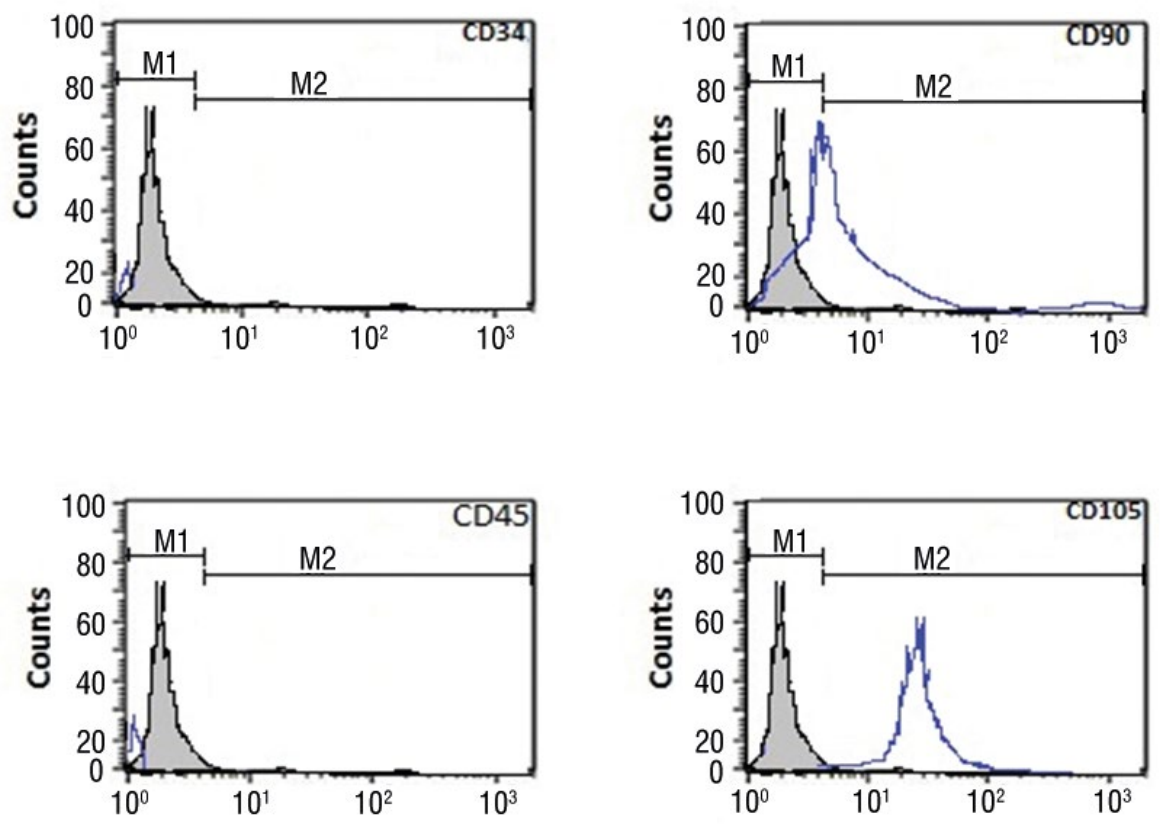

Figure 1. Flow cytometry for adipose tissue stem cells showing culture of differentiation (CD). CD 90 and CD 105 were positive and CD 34 and CD 45 were negative.

medium (DMEM, GIBCO) containing 10\% foetal bovine serum (FBS, GIBCO), 2 mM L-glutamine, penicillin, and streptomycin (all from Invitrogen) as MSCs culture media and incubated at $37^{\circ} \mathrm{C}$ in $5 \% \mathrm{CO}_{2}$. After 48 hours, non-adherent cells were removed and fresh media were added. When adherent cells were confluent, they were trypsinized, harvested, and expanded. All the experiments were performed using AD-MSCs at passage 3. Cells were identified by their morphology, adherence, and the surface markers of MSCs. They were positive for CD 34, 45, 90 and 105 and could differentiate into osteocytes and chondrocytes (Fig. 1).

\section{Labelling of MSCs with PKH26 and pre-treatment with MT}

Undifferentiated MSCs were labelled with $\mathrm{PKH} 26$, a red fluorochrome, according to the manufacturer's recommendations (Sigma, Saint Louis, Missouri, USA). MT was obtained as vial from (Sigma-Aldrich Chemical Company, St. Louis, MO, USA). One $\mathrm{mL}$ of MT was added to $40 \mathrm{~mL}$ of ethyl alcohol; the mixture was shaken for $3 \mathrm{~min}$ then $960 \mathrm{~mL}$ of saline were added. AD-MSCs were subjected to a 24-hours pre-treatment with $5 \mu \mathrm{L}$ of MT which is equivalent to $1 \mathrm{~mL}$ of the mixture, then washed three times with phosphate buffer saline (PBS) (Sigma, USA) for complete removal of the hormone from the cell suspension.

\section{Experimental animals}

A total of 40 adult male Sprague Dawley albino rats, weighing 150-200 g, were used in the current work. Rats were obtained from the animal house of faculty of medicine, Cairo University and maintained according to the standard guidelines of the Institutional Animal Care and Use Committee and after the Institutional Review Board approval. The experiment proposal was approved by the Ethics Committee, Faculty of Medicine, Cairo University. Rats were allowed to acclimatize for 2 weeks before the experiment. They were housed in cages under normal light/dark periods and fed a standard food and water ad libitum.

\section{Experimental design}

The experimental rats were divided into the following groups:

- Group I (Control, $\mathbf{n}=10$ ): the rats received a single intraperitoneal injection of $1 \mathrm{~mL}$ of $\mathrm{Na}$ -Citrate solution and a single intravenous injection of $0.5 \mathrm{~mL}$ PBS into the tail vein;

- Group II (STZ group, $\mathbf{n}=10$ ): rats were fasted for 4 hours. A single dose of $1 \mathrm{~mL} \mathrm{STZ-Na-Citrate}$ solution, equivalent to $50 \mathrm{mg} / \mathrm{kg}$ bodyweight STZ (Sigma-Aldrich Chemical Company, St. Louis, Missouri, USA) was injected intraperitoneally. Three days after the injection, the rats were tested for hyperglycaemia. They were diagnosed diabetic 
when random blood glucose level was higher than $220 \mathrm{mg} / \mathrm{dL}$;

- Group III (STZ + AD-MSCs, $n=10$ ): induction of diabetes was performed as in group II. The rats then received a single intravenous injection of AD-MSCs $\left(1 \times 10^{6}\right)$ diluted in $0.5 \mathrm{~mL}$ of PBS into the tail vein;

- Group IV (STZ + MT pre-treated AD-MSCs, $n=10$ ): induction of diabetes was performed as in group II. The rats then received a single intravenous injection of MT pre-treated AD-MSCs $\left(1 \times 10^{6}\right)$ diluted in $0.5 \mathrm{~mL}$ of PBS into the tail vein. The health status of the stem cells treated rats was stable. On the other hand, diabetic rats were worsening overtime but there was no mortality among all groups, especially untreated diabetic rats and this was probably due to the shortness of the time of the study. Blood samples were collected by retro-orbital plexus technique using capillary glass tubes. Random blood glucose levels were detected using a glucometer (ACON Laboratories, Inc., USA). Blood glucose was measured 3 days following STZ injection and at the end of the study on the $28^{\text {th }}$ day. Enzyme-Linked Immunosorbent Assay (ELISA) study was implemented to detect the levels of the following:

- Fasting serum insulin level: it was determined using the Ultra-Sensitive Mouse Insulin ELISA Kit (Crystal Chem);

- Interleukin 17 (IL-17): it is a pro-inflammatory (promoting inflammation) cytokine produced by T-helper 17 lymphocytes. It was detected using ELISA kit (Rat IL-17A Platinum ELISA; eBioscience, Inc.) following the manufacturer's protocols. Detection limit was $1 \mathrm{pg} / \mathrm{mL}$;

- Interleukin 10 (IL-10): it is a cytokine with potent anti-inflammatory properties produced primarily by monocytes and to lesser extent by lymphocytes. IL-10 plays a central role in limiting the host immune response to injury. It was detected using Mouse IL-10 ELIZA Kit (Catalogue M1000, R\&D Systems, Minneapolis, MN), according to the manufacturer's instructions. Detection limit was $4 \mathrm{pg} / \mathrm{mL}$.

At the $28^{\text {th }}$ day, the animals were weighted and sacrificed by overdose of pentobarbital; $40 \mathrm{mg} / \mathrm{kg}$ body weight intraperitoneally. Dissection and excision of the pancreas were done. Half of each pancreatic specimen of each rat was fixed in $10 \%$ formaldehyde in PBS at $4^{\circ} \mathrm{C}$ and was processed for paraffin blocks.
Paraffin sections of 5 microns were stained with haematoxylin and eosin stain for routine histological examination.

\section{Immunohistochemical study}

Paraffin sections were blocked with $1.5 \%$ normal rat serum in PBS. Then the sections were incubated for $45 \mathrm{~min}$. at room temperature and then incubated with the following primary antibodies for 30 minutes:

- proliferating cell nuclear antigen (PCNA): a cofactor of DNA polymerase- $\delta$ needed for DNA replication, DNA repair and chromatin remodelling. Positive reactions, indicating nuclear regeneration, were detected by rabbit polyclonal IgG (SC-7907, $200 \mu \mathrm{g} / \mathrm{mL}$, dilution 1:50, Santa Cruz Biotechnology, USA);

- caspase-3 antibody: caspase-3 is a fundamental mediator of apoptosis; programmed cell death. It was detected using anti-caspase-3 mouse monoclonal primary antibody (Dako Company, Cairo, Egypt. Catalogue No. IMG-144A at a dilution 1/200). The slides were rinsed in PBS (3 times, 2 min each), incubated with two drops of biotinylated secondary antibody for each section, for $20 \mathrm{~min}$, then rinsed with PBS. Two drops of substrate chromogen (DAB) mixture was applied for 5 min then rinsed with distilled water. Slides were counterstained with haematoxylin, then dehydrated and mounted.

\section{Fluorescent microscopic study}

Pancreatic sections were dipped in xylol solution to be examined unstained using Olympus IX81 Fluorescence Motorized Phase Contrast Microscope Unit8 to detect the PKH26-labelled AD-MSCs. They appeared as orange to red spots in the pancreatic tissue.

\section{Histomorphometric study}

The slides prepared for immunohistochemical studies (caspase-3 and PCNA) were assessed quantitatively. Ten non-overlapping microscopic fields were picked randomly from each slide at a magnification power of $\times 400$ and examined using "Leica Qwin 500 C" image analyser computer Ltd. (Cambridge England) within the standard measuring frame of a known area equal to $11694 \mu \mathrm{m}^{2}$. The area per cent of caspase-3 reactions, in the cytoplasm of the cells of islets of Langerhans, was measured. PCNA proliferation index was calcu- 
Table 1. Mean \pm standard deviation of the body weight, fasting blood glucose, fasting serum insulin among the studied groups

\begin{tabular}{lcccc}
\hline & Group I (Control) & Group II (STZ) & Group III (STZ + AD-MSCs) & Group IV (STZ + MT + AD-MSCs) \\
\hline Body weight [g] & $210.01 \pm 11$ & $84 \pm 10^{\mathrm{a}}$ & $134.5 \pm 18.7^{\mathrm{ab}}$ & $176.0 \pm 14.2^{\mathrm{abc}}$ \\
Fasting blood glucose [mg/dL] & $100 \pm 7.7$ & $294 \pm 20^{\mathrm{a}}$ & $170.4 \pm 4.56^{\mathrm{ab}}$ & $135.9 \pm 2.29^{\mathrm{abc}}$ \\
Fasting serum insulin $[\mu \mathrm{lU} / \mathrm{mL}]$ & $2.93 \pm 0.23$ & $1.06 \pm 0.10^{\mathrm{a}}$ & $1.70 \pm 0.10^{\mathrm{ab}}$ & $2.59 \pm 0.09^{\mathrm{abc}}$ \\
IL-17 [pg/mL] & $23.3 \pm 1.71$ & $162.2 \pm 7.54^{\mathrm{a}}$ & $93.4 \pm 5.96^{\mathrm{ab}}$ & $50.9 \pm 8.26^{\mathrm{abc}}$ \\
IL-10 [pg/mL] & $130.6 \pm 2.34$ & $46.3 \pm 2.25^{\mathrm{a}}$ & $88.2 \pm 5.88^{\mathrm{ab}}$ & $111.7 \pm 9.29^{\mathrm{abc}}$ \\
\hline
\end{tabular}

${ }^{a}$ significant to group I; 'bignificant to group II; ' significant to group III (p < 0.05); IL — interleukin; STZ — streptozotocin; AD-MSCs — adipose tissue-derived mesenchymal stem cells; MT - melatonin

lated as the percentage of PCNA positively labelled nuclei to the total number of nuclei per field in the islets of Langerhans cells and the area per cent of PKH26 labelled AD-MSCs and MT pre-treated AD-MSCs were measured in groups III and IV.

\section{Statistical study}

The data of the present study were tabulated and statistically analysed using an IBM personal computer with Statistical Package of Social Science (SPSS) version 22 (SPSS, Inc., Chicago, Illinois, USA). Quantitative data were presented in the form of mean $(\bar{x})$ and standard deviation (SD). ANOVA test of significance was used for comparison between groups having quantitative variables. $P$ value of $<0.05$ was considered statistically significant.

\section{RESULTS}

\section{Biochemical results}

Group II (STZ) showed significant statistical decrease in the mean body weight and fasting serum insulin compared to group I (Control) at the $28^{\text {th }}$ day. A significant statistical decrease in both parameters in groups III (STZ + AD-MSCs) and IV (STZ + MT + AD-MSCS) compared to group I (Control) was detected with a significant statistical increase in both groups (III and IV) compared to group II (STZ). In addition, a significant statistical increase was found in the body weight and fasting serum insulin in group IV (STZ + MT + AD-MSCs) compared to group III (STZ + AD-MSCs) $(p<0.05)$ (Table 1). Concerning the mean fasting blood glucose level, a significant statistical increase was shown in groups II (STZ), III (STZ+AD-MSCS) and IV (STZ + MT + AD-MSCs) compared to group I (Control). While a significant statistical decrease was revealed in groups III (STZ + AD-MSCs) and IV (STZ + MT + AD-MSCs) compared to group II (STZ) which was significantly decreased in group IV
(STZ + MT + AD-MSCs) more than group III (STZ + AD-MSCs) $(p<0.05)$ (Table 1).

Regarding the IL-17, groups II (STZ), III, (STZ + AD-MSCs) and IV (STZ + MT + AD-MSCs) showed a significant statistical increase compared to group I (Control). There was a significant statistical decrease in groups III (STZ + AD-MSCs) and IV (STZ + MT + AD-MSCs) compared to group II (STZ) which also was significantly decreased in group IV (STZ + MT + AD-MSCs) more than group III (STZ + AD-MSCs). IL-10 cytokine was found significantly decreased in groups II (STZ), III (STZ + AD-MSCs) and IV (STZ + MT + AD-MSCs) compared to group I (Control). There was a significant statistical increase in groups III (STZ + AD-MSCs) and IV (STZ + MT + AD-MSCs) compared to group II (STZ) which also was significantly increased in group IV (STZ + MT + AD-MSCs) more than group III (STZ + AD-MSCs) $(p<0.05)$ (Table 1).

\section{Haematoxylin and eosin study}

The pancreatic specimens of the rats of group I (Control group) revealed normal pancreatic histological architecture. Well-defined and lightly stained islets of Langerhans were surrounded by classical pancreatic acini. Islets of Langerhans contained a large number of islet cells with vesicular nuclei and prominent nucleoli (Fig. 2A, B). The specimens of the experimental rats of group II (STZ treated group) showed ill-defined shrunken islets of Langerhans containing fewer islet cells with intracytoplasmic vacuolations and surrounded by distorted acini and interacinar dilated blood capillaries. Empty spaces appeared in the islets denoting necrosis and complete cellular loss (Fig. 2C, D).

The pancreatic specimens of the rats of group III (STZ + AD-MSCs treated group) showed some improvement in the islets of Langerhans with a larger number of islet cells. The islets were surrounded by 

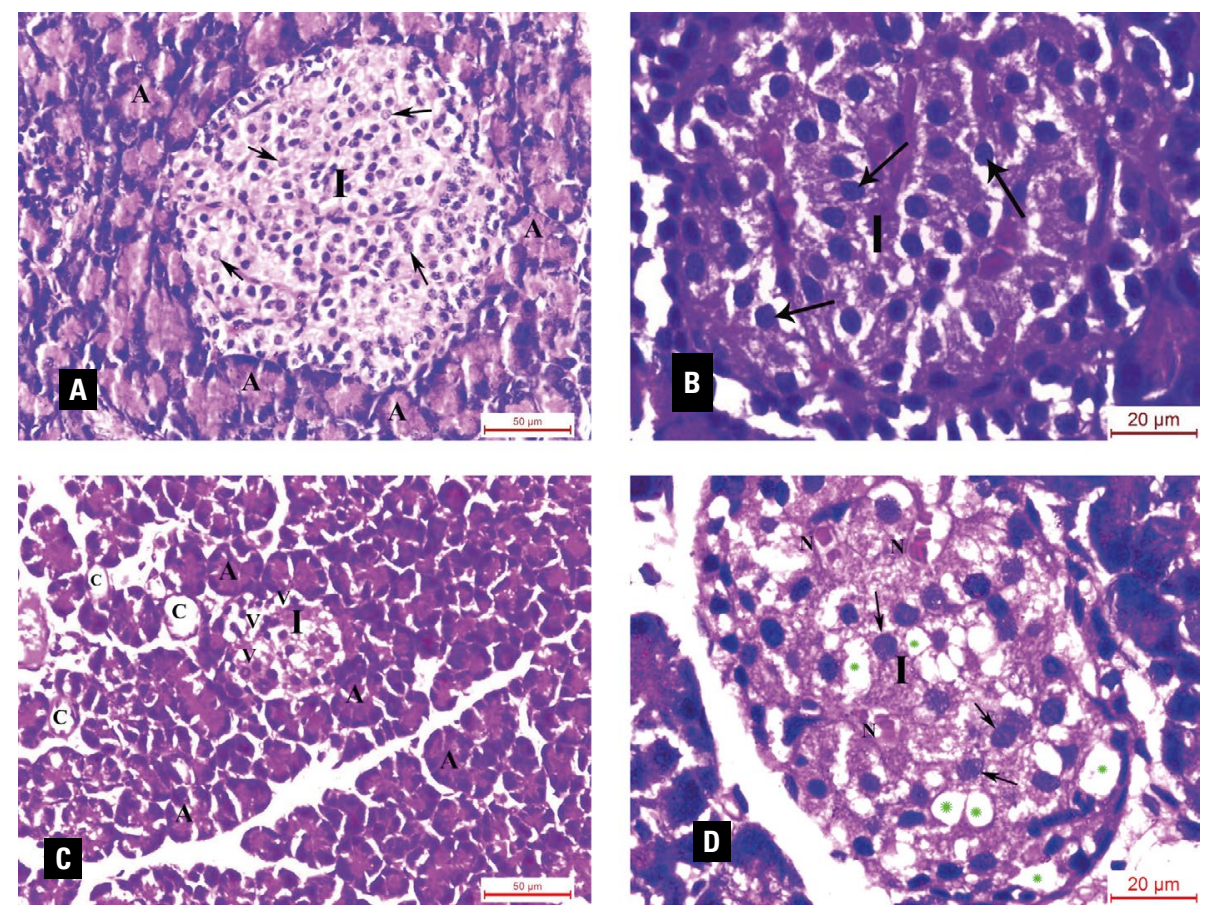

Figure 2. Photomicrographs of the pancreatic specimens of the rats; A, B. Group I (Control) showing well-defined lightly stained islets of Langerhans (I) with regular outline containing a large number of islet cells with vesicular nuclei and prominent nucleoli (arrows). The islets are surrounded by many pancreatic acini (A) having regular outline; C, D. Group II (STZ) showing ill-defined and shrunken islets of Langerhans (I) containing a fewer islet cells and surrounded by distorted acini (A). Some islet cells show intracytoplasmic vacuolations (v). Some interacinar blood capillaries (C) appear dilated. Some islet cells appear normal (arrows), some others show necrosis (N). The islets show many empty spaces (asterisk); haematoxylin and eosin, A, C ×400; B, D × 1000).

many regular pancreatic acini. Few islet cells revealed pyknotic nuclei and multiple empty spaces (Fig. 3A, B). Group IV (STZ + MT treated AD-MSCs group) showed well-defined lightly stained islets of Langerhans, with a larger number of islet cells compared to group II (STZ treated group). The islets were surrounded by regular pancreatic acini (Fig. 3C, D).

\section{Immunohistochemical study}

Positive PCNA immune reaction, in the form of brown nuclei, appeared in the islet cells of group I; the control group. However, very few islet cells of group II (STZ) exhibited PCNA reaction. Group III (STZ + AD-MSCs) revealed a moderate number of islet cells with positive PCNA reaction. Group IV (STZ + MT treated AD-MSCs) showed a larger number of brown nuclei (Fig. 4A-D). A negative caspase-3 reaction was found in the control group. Marked positive caspase- 3 reaction in the form of brown discoloration of the cellular cytoplasm was detected in the islet cells of group II (STZ). A moderate positive reaction appeared in group III (STZ + AD-MSCS) and few positive reactions were found in group IV (STZ + MT treated AD-MSCs) (Fig. 5A-D).

\section{Fluorescent microscopic study}

Homing and proliferation of the PKH26 labelled masses of AD-MSCs appeared in the pancreatic specimens of the rats of group III (STZ + AD-MSCS) and group IV (STZ + MT treated AD-MSCs) (Fig. 6A, B).

\section{Histomorphometric study}

The area per cent of caspase- 3 immuno-expression was significantly increased in groups II (STZ) and III (STZ+AD-MSCs) compared to the control group, but was non-significant in group IV (STZ + MT + AD-MSCs) compared to the control group. While, a significant statistical decrease in the area percent in groups III and IV was detected, compared to group II (STZ) with a more significant decrease in group IV (STZ + MT + AD-MSCs) than group III $(S T Z+A D-M S C s)(p<0.05)$ (Table 2). Group II (STZ) showed a significant statistical decrease in the PCNA proliferation index compared to group I (Control). However, a significant statistical increase in the index in groups III and IV; (STZ + AD-MSCs) and (STZ + MT + AD-MSCs) was detected compared to the control group (group I) and group II (STZ) with a more significant increase in group IV (STZ 

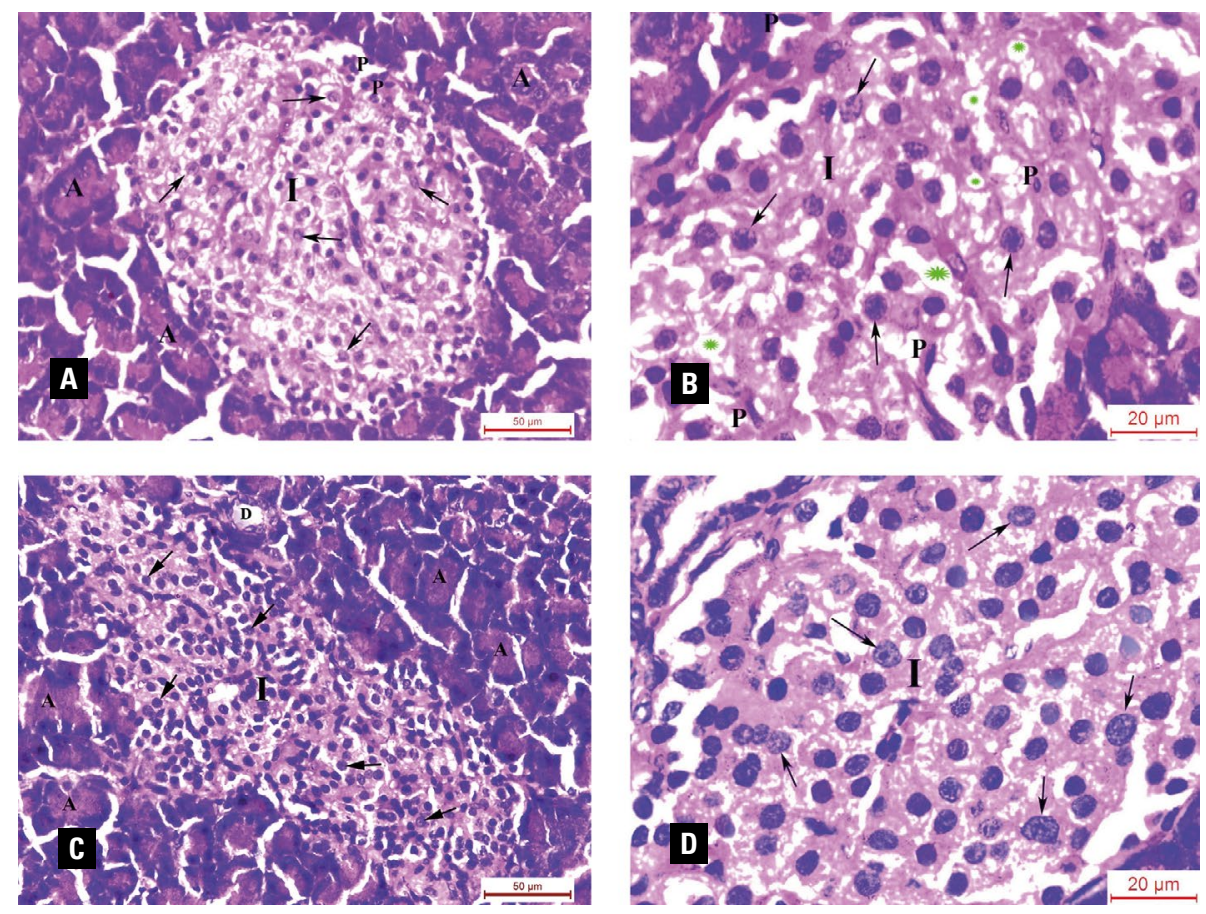

Figure 3. Photomicrographs of the pancreatic specimens of the rats; A, B. Group III (STZ + AD-MSCs) showing islets of Langerhans (I) containing a larger number of islets cells (arrows). The islet is surrounded by many pancreatic acini (A) showing regular outline. Few islets cells show pyknotic nuclei (P). The islet shows multiple empty spaces (asterisk); C, D. Group IV (STZ + MT treated AD-MSCs) showing well-defined and lightly stained islets of Langerhans (I) containing a huge number of islets cells (arrows). The islets are surrounded by many pancreatic acini (A) showing regular outline. A pancreatic duct (D) shows regular outline with regular epithelial lining; haematoxylin and eosin, $A, C \times 400 ; B, D \times 1000$.
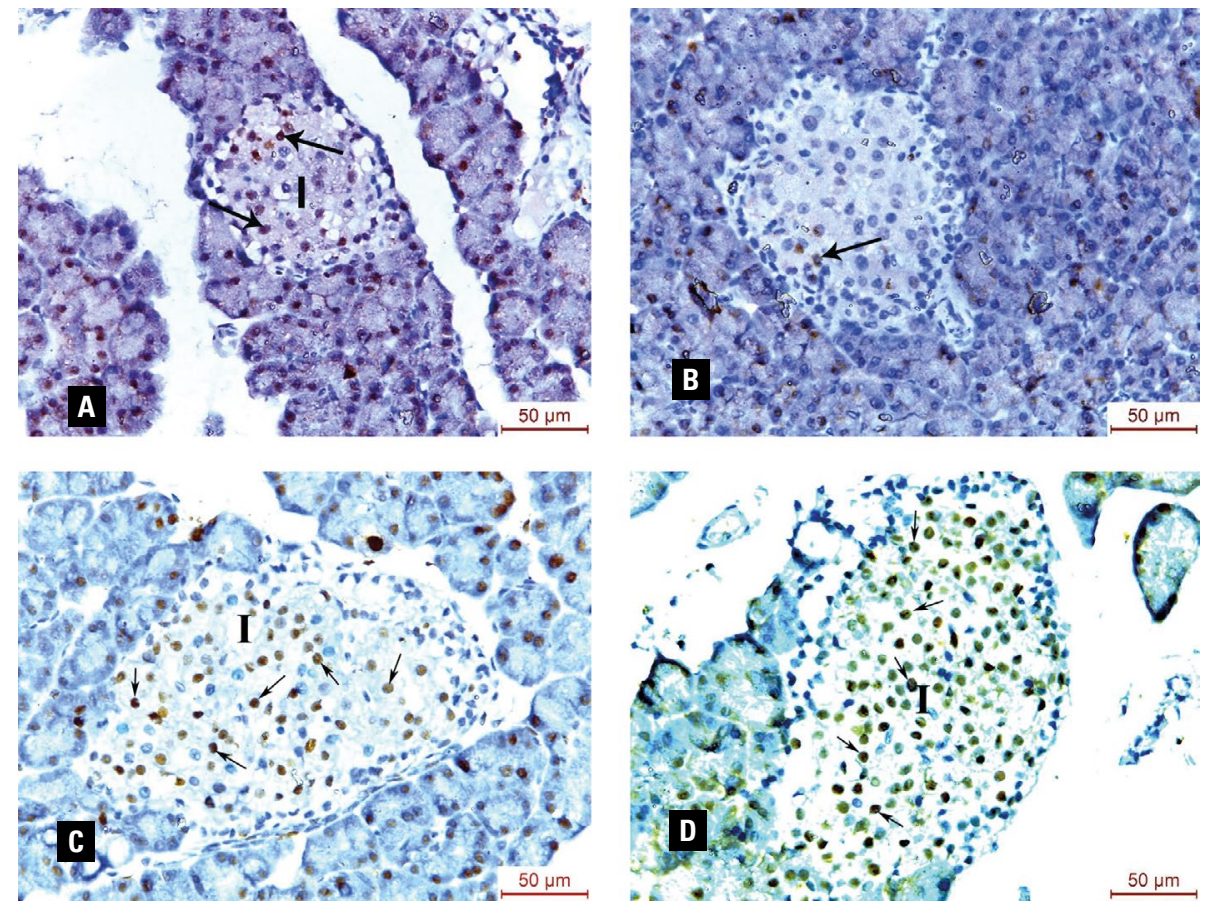

Figure 4. Photomicrographs of the islets of Langerhans (I); A. Group I (Control) showing the islet cells having brown nuclei with positive proliferating cell nuclear antigen (PCNA) reaction (arrows); B. Group II (STZ) showing very few islet cells exhibiting PCNA reaction; C. Group III (STZ + AD-MSCs) showing moderate number of islet cells having brown nuclei (arrows); D. Group IV (STZ + MT treated AD-MSCs) showing larger number of brown nuclei (arrows); PCNA $\times 400$. 

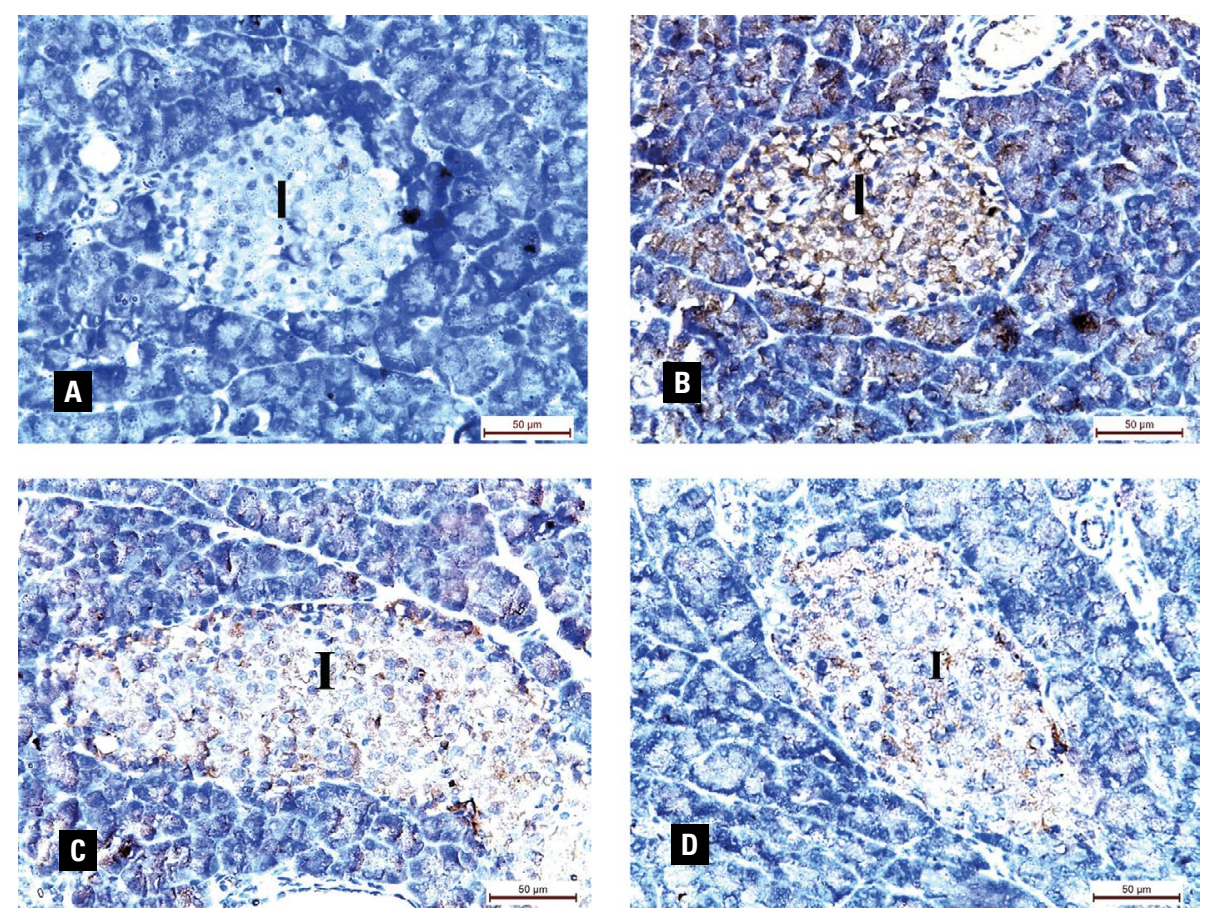

Figure 5. Photomicrographs of the islet of Langerhans (I); A. Group I (Control) showing the islet cells with negative caspase-3 reaction in their cytoplasm; B. Group II (STZ) showing the islet cells with marked positive caspase-3 reaction in the form of brown discolouration of their cytoplasm; C. Group III (STZ + AD-MSCs) showing the islet cells with moderate positive caspase-3 reaction; D. Group IV (STZ + MT treated AD-MSCs) showing few positive caspase- 3 reaction the islet cells; caspase- $3 \times 400$.
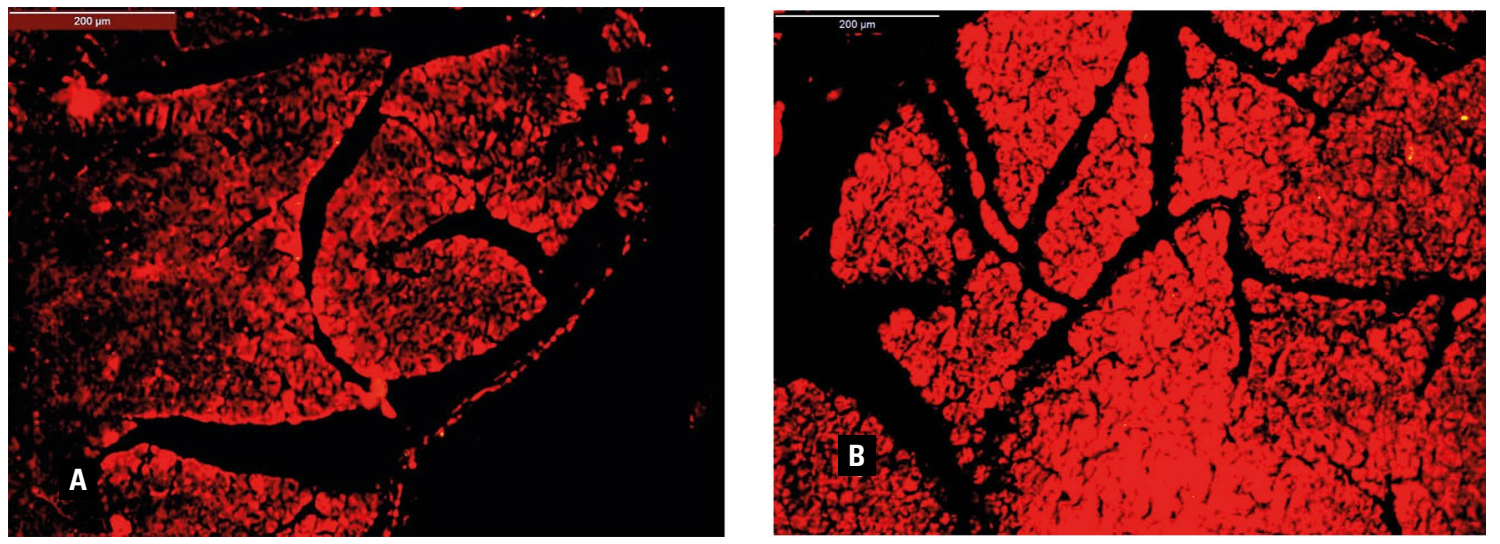

Figure 6. Photomicrographs of the pancreatic specimens of the rats; A. Group III (STZ+AD-MSCs); B. Group IV (STZ + MT treated AD-MSCs) that show homing and proliferation of the PKH26-labelled masses of AD-MSCs inside the pancreatic lobules and interlobular regions; PKH26 $\times 100$.

Table 2. Mean \pm standard deviation of the area per cent of caspase-3 immuno-expression, PKH26 labelled adipose tissue-derived mesenchymal stem cells (AD-MSCs) and proliferating cell nuclear antigen (PCNA) proliferation index among the studied groups

\begin{tabular}{lcccc}
\hline & Group I (Control) & Group II (STZ) & Group III (STZ + AD-MSCs) & Group IV (STZ + MT + AD-MSCs) \\
\hline Caspase-3 & $0.02 \pm 0.02$ & $55.24 \pm 12^{\mathrm{a}}$ & $16.5 \pm 1.47^{\mathrm{ab}}$ & $1.04 \pm 0.97^{\mathrm{bc}}$ \\
PCNA index & $10 \pm 1.65$ & $0.02 \pm 0.01^{\mathrm{a}}$ & $41.4 \pm 2.59^{\mathrm{ab}}$ & $78.1 \pm 3.2^{\mathrm{bac}}$ \\
PKH26 & - & - & $26.6 \pm 9.02$ & $73.1 \pm 7.28^{\mathrm{c}}$ \\
\hline
\end{tabular}

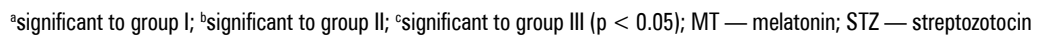


$+\mathrm{MT}+\mathrm{AD}-\mathrm{MSCs}$ ) compared to group III (STZ + AD-MSCs) $(p<0.05)$ (Table 2$)$. Group IV (STZ + $\mathrm{MT}+\mathrm{AD}-\mathrm{MSCs})$ revealed a significant statistical increase in the area per cent of PKH26 labelled MSCs compared to group III (STZ + AD-MSCs) $(p<0.05)$ (Table 2).

\section{DISCUSSION}

Mesenchymal stem cells represent a promising tool in the treatment of diabetes mellitus (DM) and they were applied successfully for this purpose. The present work was carried out to throw more light on the effects of AD-MSCs on the islets of Langerhans in STZ-induced diabetic rats as well as the possible enhancing role of adding MT. In the current work, AD-MSCs-treated groups revealed improvement of STZ-induced damage of the pancreas indicating a potential regenerative role of MSCs. These findings are consistent with previous studies that demonstrated low blood glucose levels, increased serum insulin and resuming the rats' body weight [1]. Several studies have demonstrated the mechanisms by which MSCs could improve STZ-induced islet cells damage. According to Ullah et al. [22], the initial step in MSCs strategy is the "homing" phenomenon. They found that the systemically administered MSCs migrated to the site of injury, attributing it to the chemo-attraction mediated by cell surface receptors such as the chemokine receptors. The role of the cytoskeleton receptors, such as integrin and selectin has been also suggested by Nawaz et al. [15] to mediate MSCs and endothelial interaction, facilitating the MSCs entry to the site of injury. Teo et al. [20] added that the vascular cell adhesion molecule-1 (VCAM-1) and G-protein-coupled receptor signalling contributed to the process of homing. The homing phenomenon of MSCs was demonstrated in the present work by the significant increase in the area percent of PKH-labelled MSCs in the pancreatic lobules and interlobular regions, especially in group IV (STZ + MT pretreated AD-MSCs). Another suggested mechanism of the MSCs promising role is their differentiation into $\beta$-cells of islets of Langerhans [16]. Chandra et al. [5], reported that AD-MSCs could differentiate into IPCs in STZ-induced diabetes enhancing their treatment. Volarevic et al. [23] suggested another mechanism of MSCs in improving STZ induced diabetes which is the ability of MSCs to promote the regeneration and proliferation of endogenous injured pancreatic cells even if they did not home in the islets of Langerhans through the production of a variety of cytokines and growth factors. Zang et al. [25] reported that MSCs create a microenvironment that allows endogenous islets cells to proliferate and regain their normal function. They suggested a number of factors, such as the vascular endothelial growth factor (VEGF)-alpha, insulin-like growth factor (IGF)-1, platelet-derived growth factor (PDGF) and angiopoietin-1, to play an essential role in the process of islet cells regeneration. In the present work, the significant increase of PCNA proliferation index in the islet cells together with the significant increase of the anti-inflammatory marker; IL-10 could support the mechanism of the regenerative effect of AD-MSCs on STZ injured islet cells. These findings are in agreement with Zhang et al. [26] and Omar and Aboulkhair [16] who reported that AD-MSCs secrete trophic and angiogenic factors. Another mechanism of MSCs in improving STZ induced DM is the protection of endogenous pancreatic islet cells against inflammation and apoptosis through their immunoregulatory abilities as suggested by Bloom et al. [4] who supposed that MSCs have immunosuppressive abilities and could suppress the proliferation of T lymphocytes, the main effectors in autoimmune diseases like type one DM, and inhibit dendritic cell differentiation [27]. Moreover, MSCs inhibit the proliferation, maturation and antibody secretion of B lymphocytes [24]. Zang et al. [25] also found that MSCs reduced apoptosis among islets cells and increased their viability following the co-culture of both cells for 48 hours. Cho et al. [6] reported that the cytokine analysis of MSCs derived islets cells revealed increased expression of anti-inflammatory cytokines like TGF- $\beta$ and TNF- $\alpha$, lower levels of pro-inflammatory cytokines, reduced levels of reactive oxygen species, downregulated caspase-3 and caspase-8 confirming the MSCs' anti-apoptotic properties. This assumption was supported by the findings of the current study where AD-MSCs treated groups showed a significant decrease in the area per cent of caspase-3 immuno-expression, significant decrease in IL-17 compared to STZ group denoting decreased inflammation and protection of islet cells against apoptosis, especially group IV (STZ + MT treated AD-MSCs).

Mortezaee et al. [13] reported that MSCs highly expressed MT1 and MT2 receptors on their surface and that MT produced its effects on MSCs via receptor-dependent and receptor-independent mechanisms by which the antioxidant enzymes such as catalase and superoxide dismutase-1 were overex- 
pressed, therefore increasing the MSCs resistance to hydrogen peroxide dependent apoptosis and increasing their therapeutic values [28]. These findings support the results of the current work that revealed improvement in all biochemical and histopathological parameters in the group of AD-MSCs enriched with MT, which confirm the enhancing role of MT on the proliferative and regenerative activities of AD-MSCs on the islet cells. Although, in the present work, the AD-MSCs treated groups showed significant improvement, but still not reaching the control level. Omar and Aboulkhair [16] attributed that to the arrival of a limited small number of MSCs into the pancreas after their transplantation. Hence the attraction of the stem cells to the site of injury is mediated by stem cells' chemokines, the injured tissue ligands might be not enough to attract a sufficient amount of MSCs to the site of the lesion to restore normal conditions. El-Said et al. [7] also reported that although MSCs lowered blood glucose levels as compared to diabetic non-treated rats, after six weeks of transplantation, serum glucose levels were still higher compared to the control. They reported that treatment of diabetic rats with MSCs may be more effective in repeated doses and longer periods. Moreover, Hu and Li [9] deduced that high MT concentrations might harm the epigenetic and genetic stability of MSCs.

Clinically, this work confirms the novelty of stem cells therapy in DM with promising chances for the use of such so natural remedy for application in human. AD-MSCs could ameliorate the diabetic status of rats by improving STZ-induced disturbances in the islets of Langerhans with amelioration of hyperglycaemia and improving serum insulin level. Therefore, further investigations are recommended to determine the optimal concentration and administration time to produce the efficient regeneration and protection capabilities of MT enriched AD-MSCs for the islet cells in the near future.

\section{Limitations of the study}

An experimental work of longer duration is necessarily needed to confirm the stability of the therapeutic value and the overall improvement in the diabetic rats treated with AD-MSCs, as well as a longer time of diabetic status before the administration of the stem cells to treat a long-standing full blown picture of the DM disease. Also the need of a booster injection of AD-MSCs was not ruled out.
The cell line and the age of the obtained stem cells for treatment of diabetic rats is a major concern determining their clinical benefits. Cells might not have reached the target organ in sufficient amounts due to any vascular obstruction in the way or may me rejected due to some immunological issues.

\section{CONCLUSIONS}

From the results of the current work, it could be concluded that pretreatment of AD-MSCs with MT enhances their power of treatment and protection of the cells of the pancreatic islets of Langerhans. Further studies are needed to explore the stability of the therapeutic effects of AD-MSCs in DM as well as the possible side effects over longer experimental durations.

\section{Acknowledgements}

The authors would like to acknowledge the College of Medicine, Qassim University for the generous facilitation of the publication of this manuscript.

\section{Conflict of interest: None declared}

\section{REFERENCES}

1. Ammar HI, Sequiera GL, Nashed MB, et al. Comparison of adipose tissue- and bone marrow-derived mesenchymal stem cells for alleviating doxorubicin-induced cardiac dysfunction in diabetic rats. Stem Cell Res Ther. 2015; 6: 148, doi: 10.1186/ s13287-015-0142-x, indexed in Pubmed: 26296856.

2. Basoli V, Santaniello S, Cruciani $S$, et al. Melatonin and vitamin $D$ interfere with the adipogenic fate of adipose-derived stem cells. Int J Mol Sci. 2017; 18(5), doi: 10.3390/ ijms18050981, indexed in Pubmed: 28475114.

3. Berebichez-Fridman R, Montero-Olvera PR. Sources and clinical applications of mesenchymal stem cells: state-ofthe-art review. Sultan Qaboos Univ Med J. 2018; 18(3): e264-e277, doi: 10.18295/squmj.2018.18.03.002, indexed in Pubmed: 30607265.

4. Bloom DD, Centanni JM, Bhatia N, et al. A reproducible immunopotency assay to measure mesenchymal stromal cell-mediated T-cell suppression. Cytotherapy. 2015; 17(2): 140-151, doi: 10.1016/j.jcyt.2014.10.002, indexed in Pubmed: 25455739.

5. Chandra V, Swetha G, Muthyala S, et al. Islet-like cell aggregates generated from human adipose tissue derived stem cells ameliorate experimental diabetes in mice. PLoS One. 2011; 6(6): e20615, doi: 10.1371/journal.pone.0020615, indexed in Pubmed: 21687731.

6. Cho J, D'Antuono M, Glicksman M, et al. A review of clinical trials: mesenchymal stem cell transplant therapy in type 1 and type 2 diabetes mellitus. Am J Stem Cells. 2018; 7(4): 82-93, indexed in Pubmed: 30510843

7. El-Said KS, Ezz AA, El Ba, et al. The potential role of male bone marrow mesenchymal stem cells of diabetic female rats. Diabetes Manag. 2018; 8(6): 137-146. 
8. Hashemian SJ, Kouhnavard M, Nasli-Esfahani E. Mesenchymal stem cells: rising concerns over their application in treatment of type one diabetes mellitus. J Diabetes Res. 2015; 2015: 675103, doi: 10.1155/2015/675103, indexed in Pubmed: 26576437.

9. Hu C, Li L. Melatonin plays critical role in mesenchymal stem cell-based regenerative medicine in vitro and in vivo. Stem Cell Res Ther. 2019; 10(1): 13, doi: 10.1186/s13287018-1114-8, indexed in Pubmed: 30635065.

10. Huang $L$, Wang $G$. The effects of different factors on the behavior of neural stem cells. Stem Cells Int. 2017; 2017: 9497325 , doi: $10.1155 / 2017 / 9497325$, indexed in Pubmed: 29358957.

11. Karaoz E, Okcu A, Ünal ZS, et al. Adipose tissue-derived mesenchymal stromal cells efficiently differentiate into insulin-producing cells in pancreatic islet microenvironment both in vitro and in vivo. Cytotherapy. 2013; 15(5): 557-570, doi: 10.1016/j.jcyt.2013.01.005, indexed in Pubmed: 23388582.

12. Luchetti F, Canonico B, Bartolini D, et al. Melatonin regulates mesenchymal stem cell differentiation: a review. J Pineal Res. 2014; 56(4): 382-397, doi: 10.1111/jpi.12133, indexed in Pubmed: 24650016.

13. Mortezaee K, Khanlarkhani N, Sabbaghziarani F, et al. Preconditioning with melatonin improves therapeutic outcomes of bone marrow-derived mesenchymal stem cells in targeting liver fibrosis induced by $\mathrm{CCl} 4$. Cell Tissue Res. 2017; 369(2): 303-312, doi: 10.1007/s00441-0172604-1, indexed in Pubmed: 28413861.

14. Moshtagh PR, Emami SH, Sharifi AM. Differentiation of human adipose-derived mesenchymal stem cell into insulin-producing cells: an in vitro study. J Physiol Biochem. 2013; 69(3): 451-458, doi: 10.1007/s13105-012-0228-1, indexed in Pubmed: 23271274.

15. Nawaz M, Fatima F, Vallabhaneni KC, et al. Extracellular vesicles: evolving factors in stem cell biology. Stem Cells Int. 2016; 2016: 1073140, doi: 10.1155/2016/1073140, indexed in Pubmed: 26649044.

16. Omar A, Aboulkhair A. Effect of bone marrow versus adipose tissue derived mesenchymal stem cells on the pancreas of streptozotocin-induced diabetes mellitus type I in adult male rats (histological study). Egypt J Histol. 2017; 40(1): 12-24, doi: 10.21608/ejh.2017.3693.

17. Pei $M$, He $F$, Wei $L$, et al. Melatonin enhances cartilage matrix synthesis by porcine articular chondrocytes. J Pineal Res. 2009; 46(2): 181-187, doi: 10.1111/j.1600079X.2008.00646.x, indexed in Pubmed: 19054299.

18. Sneddon JB, Tang Q, Stock $P$, et al. Stem cell therapies for treating diabetes: progress and remaining challenges. Cell Stem Cell. 2018; 22(6): 810-823, doi: 10.1016/j. stem.2018.05.016, indexed in Pubmed: 29859172.
19. Stehle JH, Saade A, Rawashdeh O, et al. A survey of molecular details in the human pineal gland in the light of phylogeny, structure, function and chronobiological diseases. J Pineal Res. 2011; 51(1): 17-43, doi: 10.1111/j.1600-079X.2011.00856.x, indexed in Pubmed: 21517957.

20. Teo GSL, Ankrum JA, Martinelli R, et al. Mesenchymal stem cells transmigrate between and directly through tumor necrosis factor- $\alpha$-activated endothelial cells via both leukocyte-like and novel mechanisms. Stem Cells. 2012; 30(11): 2472-2486, doi: 10.1002/stem.1198, indexed in Pubmed: 22887987.

21. Thakkar UG, Trivedi HL, Vanikar AV, et al. Insulin-secreting adipose-derived mesenchymal stromal cells with bone marrow-derived hematopoietic stem cells from autologous and allogenic sources for type 1 diabetes mellitus. Cytotherapy. 2015; 17(7): 940-947, doi: 10.1016/j. jcyt.2015.03.608, indexed in Pubmed: 25869301.

22. Ullah M, Liu DD, Thakor AS. Mesenchymal stromal cell homing: mechanisms and strategies for improvement. iScience. 2019; 15: 421-438, doi: 10.1016/j.isci.2019.05.004, indexed in Pubmed: 31121468.

23. Volarevic V, Al-Qahtani A, Arsenijevic N, et al. Interleukin-1 receptor antagonist (IL-1Ra) and IL-1 Ra producing mesenchymal stem cells as modulators of diabetogenesis. Autoimmunity. 2010; 43(4): 255-263, doi: 10.3109/08916930903305641, indexed in Pubmed: 19845478.

24. Wang Y, Chen X, Cao W, et al. Plasticity of mesenchymal stem cells in immunomodulation: pathological and therapeutic implications. Nat Immunol. 2014; 15(11): 1009-1016, doi: 10.1038/ni.3002, indexed in Pubmed: 25329189.

25. Zang Li, Hao H, Liu J, et al. Mesenchymal stem cell therapy in type 2 diabetes mellitus. Diabetol Metab Syndr. 2017; 9: 36, doi: 10.1186/s13098-017-0233-1, indexed in Pubmed: 28515792.

26. Zhang HT, Liu ZL, Yao XQ, et al. Neural differentiation ability of mesenchymal stromal cells from bone marrow and adipose tissue: a comparative study. Cytotherapy. 2012; 14(10): 1203-1214, doi: 10.3109/14653249.2012 .711470, indexed in Pubmed: 22909277.

27. Zhang $Y, G e X H$, Guo XJ, et al. Bone marrow mesenchymal stem cells inhibit the function of dendritic cells by secreting galectin-1. Biomed Res Int. 2017; 2017: 3248605, doi: $10.1155 / 2017 / 3248605$, indexed in Pubmed: 28713822.

28. Zhao $L$, Hu C, Zhang P, et al. Melatonin preconditioning is an effective strategy for mesenchymal stem cellbased therapy for kidney disease. J Cell Mol Med. 2020; 24(1): 25-33, doi: $10.1111 / \mathrm{jcmm} .14769$, indexed in Pubmed: 31747719. 